\title{
Incidence and mortality of laryngeal cancer in China, 2008-2012
}

\author{
Yuqin Liu' ${ }^{1}$, Qin Zhao', Gaoheng Ding'1, Yitong Zhu' ${ }^{1}$, Wenying Li' ${ }^{1}$, Wanqing Chen ${ }^{2}$ \\ ${ }^{1}$ Central Cancer Registry of Gansu, Gansu Cancer Hospital, Lanzhou 730050, China; ${ }^{2}$ National Cancer Center/Cancer Hospital, Chinese Academy \\ of Medical Sciences \& Peking Union Medical College, Beijing 100021, China \\ Correspondence to: Wanqing Chen. National Cancer Center/Chinese Academy of Medical Sciences \&Peking Union Medical College, Beijing 100021, \\ China. Email: chenwq@cicams.ac.cn..
}

\begin{abstract}
Objective: To analyze the incidence and mortality rates of laryngeal cancer in China from 2008 to 2012.

Methods: Incident and death cases of laryngeal cancer were retrieved from the National Central Cancer Registry (NCCR) database collecting from 135 cancer registries in China during 2008-2012. The crude incidence and mortality rates of laryngeal cancer were calculated by area (urban/rural), region (eastern, middle, western), gender and age group $(0,1-4,5-9, \ldots, 85+)$. China census in 2000 and Segi's world population were applied for age standardized rates. JoinPoint (Version 4.5.0.1) model was used for time trend analysis.

Results: The crude incidence rate of laryngeal cancer was $1.86 / 100,000$ ranked the 21 st in overall cancers. The age-standardized incidence rates by China population (ASIRC) and by World population (ASIRW) were 1.22/100,000 and 1.23/100,000, respectively. The crude mortality of laryngeal cancer in China was 1.01/100,000 and it was the 21 st cause of cancer-related death in overall cancers. Both the age-standardized mortality rates by Chinese standard population (ASMRC) and by world standard population (ASMRW) were 0.63/100,000. Incidence and mortality rates of laryngeal cancer were higher in males than in females and higher in urban areas than in rural areas. Middle areas had the highest incidence and mortality rates followed by eastern and western areas. Incidence and mortality rates of laryngeal cancer retained low level before age of 40 years old but increased greatly after and peaked in age group of 75 . Incidence showed significant down trends in recent 10 years by $1.27 \%$ annually [ $95 \%$ confidence interval (95\% CI): $-2.2 \%,-0.3 \%$. Mortality declined in females sharply by $5.18 \%$ per year although stable in males and both sexes combined.
\end{abstract}

Conclusions: Appropriate targeted prevention, early detection and treatment programs should be carried out to control the local burden of laryngeal cancer.

Keywords: Laryngeal cancer; incidence; mortality; China

Submitted Feb 28, 2018. Accepted for publication May 10, 2018.

doi: 10.21147/j.issn.1000-9604.2018.03.02

View this article at: https://doi.org/10.21147/j.issn.1000-9604.2018.03.02

\section{Introduction}

Laryngeal cancer is one of common malignant tumors in the head and neck, the incidence of laryngeal cancer accounts for $1 \%-5 \%$ of all malignant tumors, and ranks third in otolaryngology cancer, behind nasopharyngeal carcinoma and paranasal sinus cancer (1). According to GLOBOCAN 2012, the global incidence and mortality rate of laryngeal cancer was $2.1 / 100,000$ and $1.1 / 100,000$, respectively. The incidence and mortality rate of laryngeal cancer in China was $1.1 / 100,000$ and $0.7 / 100,000$ respectively, which was ranked the 21 st incidence and the 21st cause of cancer-related death in all cancer in China (2). The epidemic characteristics of laryngeal cancer during 2008-2012 in China were analyzed to provide basic information for the control and research of laryngeal cancer.

\section{Materials and methods}

\section{Cancer registry data source}

National Central Cancer Registry (NCCR) of China 
collected cancer registration data from population-based cancer registries in China. A total of 164 cancer registries in China submitted the registration data from 2008 to 2012 and the data of 135 cancer registries were included in pooled data for analysis according to the criteria of quality control. Among the 135 cancer registries there were 56 registries located in urban areas and 79 registries located in rural areas. These cancer registries covered a population of $629,333,910$ person-years $(318,623,600$ males and $310,710,310$ females, $382,669,450$ urban areas and $246,664,460$ rural areas). The analysis data of laryngeal cancer (International Statistical Classification of Diseases and Related Health Problems 10th Revision, ICD-10 C32) including incidence, death records and annual population are derived from the database of NCCR. Furthermore, each year, rate of incidence/mortality of laryngeal cancer and the data of population during 2003-2007 were acquired from the database of NCCR.

\section{Quality control}

Cancer registry data submitted to NCCR underwent quality control based on the criteria of "Guideline for Chinese Cancer Registration" (3) and "Cancer Incidence in Five Continents Volume IX” by International Agency for Research on Cancer/International Association of Cancer Registries (IARC/IACR) (4). The validity, reliability, completeness and comparability of cancer registry data were evaluated based on a comprehensive consideration of a series of indexes including the mortality to incidence $(\mathrm{M} / \mathrm{I})$ ratio, the percentage of cases morphologically verified $(\mathrm{MV} \%)$, and the percentage of death certificateonly cases (DCO\%) (5). In the present analytical data of laryngeal cancer, the percentage of $\mathrm{MV}$ was accounted for $77.08 \%$, that of DCO $\%$ was $2.43 \%$ and the ratio of $\mathrm{M} / \mathrm{I}$ was 0.53 . In urban cancer registries, those indictors were
$80.57 \%, 1.99 \%$ and 0.49 , respectively. In rural cancer registries, the $\mathrm{MV} \%, \mathrm{DCO} \%$ and the ratio of $\mathrm{M} / \mathrm{I}$ were $68.93 \%, 3.46 \%$ and 0.67 , respectively (Table 1 ).

\section{Statistical analysis}

Cancer registration areas were classified into urban or rural areas according to the National Bureau of Statistics of the People's Republic of China. Crude cancer incidence and mortality rates of laryngeal cancer were calculated by area (urban/rural), region (eastern, middle, western), gender (male, female) and age groups ( $0-, 1-4,5-84$ by 5 years and $85+$ years), Age-standardized rates by Chinese and world were calculated using the population composition of China in 2000 and Segi's population structure as the standard population respectively. The cumulative risk of developing or dying from cancer before 75 years old (in the absence of competing causes of death) was calculated and presented as a percentage (6). The annual percentage change (APC) of the rate was calculated for time trend analysis from 2003 to 2012. SAS software (Version 9.4; SAS Institute Inc., Cary, NC, USA) and Joinpoint Regression Program (Version 4.5.0.1; Statistical Methodology and Applications Branch, Surveillance Research Program, National Cancer Institute, Rockville, MD, USA) were applied for statistic analysis. $\mathrm{P}<0.05$ was considered statistically significant.

\section{Results}

\section{Incidence of laryngeal cancer}

The new cases diagnosed with laryngeal cancer of China from 2008 to 2012 was 11,690 (10,500 for males and 1,190 for females), accounting for $0.66 \%$ of overall new cancer cases. The crude incidence rate of laryngeal cancer was

Table 1 Quality evaluation of laryngeal cancer in China, 2008-2012

\begin{tabular}{|c|c|c|c|c|c|c|c|c|c|}
\hline \multirow{2}{*}{ Year } & \multicolumn{3}{|c|}{ All } & \multicolumn{3}{|c|}{ Urban } & \multicolumn{3}{|c|}{ Rural } \\
\hline & MV\% & DCO\% & $\mathrm{M} / \mathrm{I}$ & MV\% & DCO\% & $\mathrm{M} / \mathrm{I}$ & MV\% & DCO\% & $\mathrm{M} / \mathrm{I}$ \\
\hline 2008 & 78.83 & 1.44 & 0.53 & 79.91 & 1.40 & 0.47 & 72.53 & 1.65 & 0.86 \\
\hline 2009 & 76.69 & 2.93 & 0.52 & 78.86 & 2.54 & 0.47 & 67.69 & 4.49 & 0.72 \\
\hline 2010 & 74.97 & 3.06 & 0.53 & 80.75 & 1.90 & 0.49 & 64.89 & 5.07 & 0.60 \\
\hline 2011 & 78.24 & 2.66 & 0.54 & 82.92 & 2.00 & 0.50 & 68.07 & 4.10 & 0.63 \\
\hline 2012 & 76.69 & 2.06 & 0.54 & 80.42 & 2.11 & 0.52 & 71.49 & 2.00 & 0.56 \\
\hline Total & 77.08 & 2.43 & 0.53 & 80.57 & 1.99 & 0.49 & 68.93 & 3.46 & 0.67 \\
\hline
\end{tabular}

MV\%, the percentage of cases morphologically verified; DCO\%, the percentage of death certificate-only cases; M/l, mortality to incidence ratio. 
$1.86 / 100,000$ which ranked the 21 st most common cancer in all cancer sites. The age-standardized incidence rates by China population (ASIRC) and by World population (ASIRW) were 1.22/100,000 and 1.23/100,000, respectively. The cumulative incidence rate of laryngeal cancer before 75 years old was $0.08 \%$. For males, laryngeal cancer was the 17 th most common cancer, with a crude incidence of 3.30/100,000, and the ASIRC and ASIRW were 2.24/100,000 and 2.26/100,000, respectively. For females, laryngeal cancer was the $23 \mathrm{rd}$ most common cancer, with a crude incidence of $0.38 / 100,000$, and the ASIRC and ASIRW were 0.24/100,000 and 0.24/100,000, respectively.

The incidence in urban areas was higher than that in rural areas. The crude incidence rate, ASIRC and ASIRW of laryngeal cancer were $2.15 / 100,000,1.37 / 100,000$ and $1.37 / 100,000$ in urban areas, with a cumulative rate of $0.08 \%$ (0-74 years old), and 1.40/100,000, 0.98/100,000 and $0.99 / 100,000$ in rural areas, with a cumulative rate of $0.06 \%$ ( $0-74$ years old).

The crude incidence in eastern areas $(1.89 / 100,000)$ and middle areas $(1.90 / 100,000)$ was higher than that in western areas $(1.52 / 100,000)$. However, after age adjustment the incidence in middle areas (ASIRC: 1.42/100,000, ASIRW: $1.43 / 100,000)$ was higher than that in eastern areas (ASIRC: 1.18/100,000, ASIRW: 1.19/100,000) and western areas (ASIRC: 1.18/100,000, ASIRW: 1.19/100,000). The patterns of age-standardized incidence rate in males and females were similar to that of both sexes in different areas (Table 2).

\section{Age-specific incidence of laryngeal cancer}

The age-specific incidence rate of laryngeal cancer was relatively low in age groups before 40 years old and then increased with age. The incidence rates were higher in urban areas than in rural areas among patients aged $>40$ years for males and females. The incidence rates of laryngeal cancer in middle areas were higher than that in eastern areas and western areas among patients aged $>40$ years for females and males. The incidence peaked in the $75-79$ age groups in overall areas and urban areas. In rural areas, the incidence in the $70-74$ age groups was the highest among all age groups. The incidence peaked in the $70-74,75-79,85+$ age groups in eastern areas, middle areas and western areas (Figure 1, 2).

Table 2 Laryngeal cancer incidence in China, 2008-2012

\begin{tabular}{|c|c|c|c|c|c|c|c|c|}
\hline $\begin{array}{l}\text { Geographic } \\
\text { areas }\end{array}$ & Gender & $\begin{array}{l}\text { New cases } \\
(\times 1,000)\end{array}$ & $\begin{array}{c}\text { Crude incidence } \\
\left(1 / 10^{5}\right)\end{array}$ & $\begin{array}{l}\text { Ratio } \\
(\%)\end{array}$ & $\begin{array}{l}\text { ASIRC } \\
\left(1 / 10^{5}\right)^{*}\end{array}$ & $\begin{array}{l}\text { ASIRW } \\
\left(1 / 10^{5}\right)^{\star *}\end{array}$ & $\begin{array}{c}\text { Cumulative rate } \\
0-74(\%)\end{array}$ & Rank \\
\hline & Both & 11.69 & 1.86 & 0.66 & 1.22 & 1.23 & 0.08 & 21 \\
\hline \multirow[t]{3}{*}{ All areas } & Male & 10.50 & 3.30 & 1.05 & 2.24 & 2.26 & 0.14 & 17 \\
\hline & Female & 1.19 & 0.38 & 0.15 & 0.24 & 0.24 & 0.01 & 23 \\
\hline & Both & 8.29 & 2.15 & 0.72 & 1.37 & 1.37 & 0.08 & 21 \\
\hline \multirow[t]{3}{*}{ Urban } & Male & 7.51 & 3.89 & 1.20 & 2.56 & 2.57 & 0.16 & 17 \\
\hline & Female & 0.72 & 0.38 & 0.14 & 0.22 & 0.22 & 0.01 & 23 \\
\hline & Both & 0.35 & 1.40 & 0.55 & 0.98 & 0.99 & 0.06 & 22 \\
\hline \multirow[t]{3}{*}{ Rural } & Male & 0.30 & 2.38 & 0.80 & 1.71 & 1.73 & 0.11 & 17 \\
\hline & Female & 0.47 & 0.39 & 0.18 & 0.26 & 0.27 & 0.01 & 22 \\
\hline & Both & 8.35 & 1.89 & 0.64 & 1.18 & 1.19 & 0.07 & 21 \\
\hline \multirow[t]{3}{*}{ Eastern areas } & Male & 7.64 & 3.42 & 1.05 & 2.21 & 2.23 & 0.14 & 17 \\
\hline & Female & 0.71 & 0.32 & 0.12 & 0.19 & 0.19 & 0.01 & 23 \\
\hline & Both & 2.50 & 1.90 & 0.75 & 1.42 & 1.43 & 0.09 & 22 \\
\hline \multirow[t]{3}{*}{ Middle areas } & Male & 2.12 & 3.13 & 1.11 & 2.42 & 2.44 & 0.15 & 15 \\
\hline & Female & 0.39 & 0.60 & 0.27 & 0.44 & 0.43 & 0.02 & 22 \\
\hline & Both & 0.83 & 1.52 & 0.62 & 1.18 & 1.19 & 0.07 & 22 \\
\hline \multirow[t]{2}{*}{ Western areas } & Male & 0.75 & 2.68 & 0.93 & 2.15 & 2.18 & 0.12 & 16 \\
\hline & Female & 0.09 & 0.32 & 0.16 & 0.23 & 0.23 & 0.01 & 23 \\
\hline
\end{tabular}

*, age-standardized incidence rate by Chinese standard population in 2000; ${ }^{\star \star}$, age-standardized incidence rate by Segi's population. 


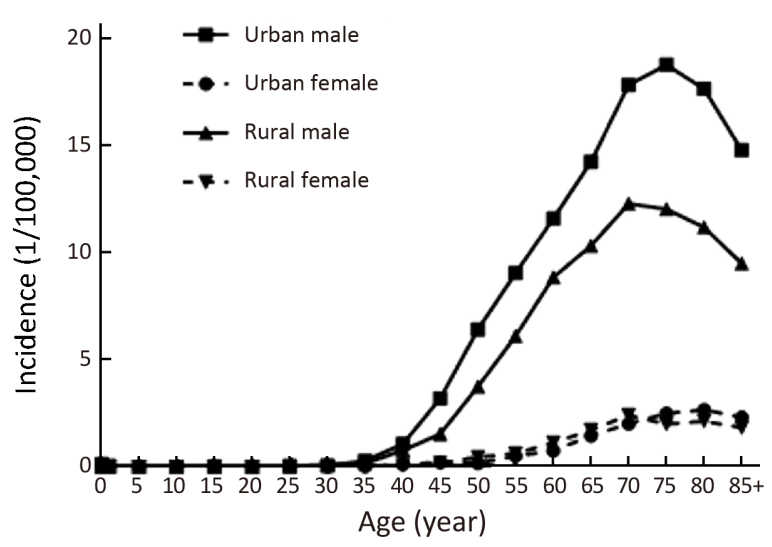

Figure 1 Age-specific incidence of laryngeal cancer in urban and rural areas in China, 2008-2012.

\section{Incidence trend of laryngeal cancer}

There was a decreased trend in the incidence of laryngeal cancer from 2003 to 2012. Compared with 2003, the incidence rate decreased by $12.75 \%$ (8.52\% for males and $50.00 \%$ for females, $14.72 \%$ for urban areas and $7.22 \%$ for rural areas). The incidence rate decreased fast in females than in males and in urban areas than in rural areas. In ten years during 2003-2012, the APC of the incidence rate in both sexes in China was $-1.27 \%$ [ $95 \%$ confidence interval (95\% CI): $-2.2 \%,-0.3 \%]$. The APC was $-7.09 \%$ (95\% CI: $-10.3 \%,-3.8 \%)$ in females and in males that was $-0.60 \%$ (95\% CI: $-1.6 \%, 0.4 \%)$. In urban areas, the decrease of incidence rate of laryngeal cancer in females was statistically significant (APC: $-8.43 \%$; 95\% CI: $-12.2 \%$,

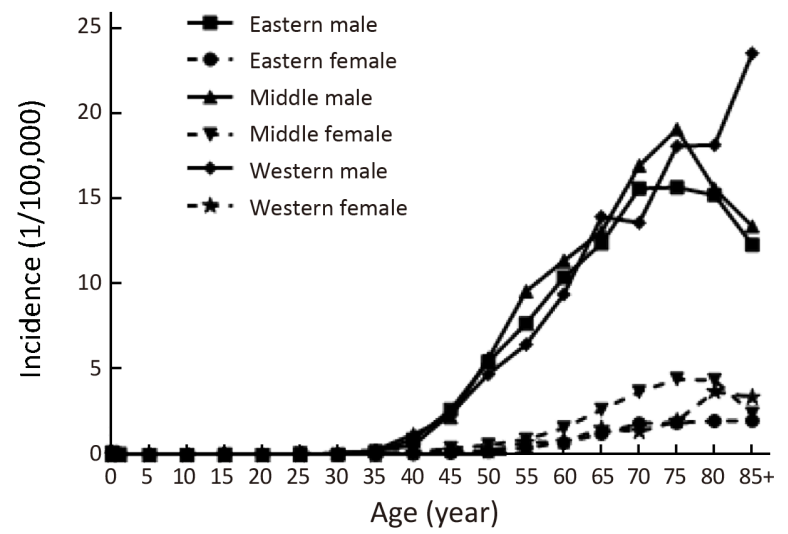

Figure 2 Age-specific incidence of laryngeal cancer in different regions in China, 2008-2012.

$-4.4 \%)(\mathrm{P}<0.05)$. The APCs of that in total and in males were $-1.38 \%$ (95\% CI: $-2.9 \%, 0.2 \%)$ and $-1.01 \%(95 \%$ CI: $-2.1 \%, 0.1 \%)$, respectively, and the change of incidence rate of laryngeal cancer was not statistically significant. In rural areas, the APCs of the rates in total, males and females were $-0.36 \%$ (95\% CI: $-2.3 \%, 1.6 \%),-0.38 \%$ (95\% CI: $-2.1 \%, 1.4 \%)$ and $-1.55 \%(95 \% \mathrm{CI}:-5.9 \%$, $3.0 \%)$, respectively, and the time change trend of the rate was not statistically significant (Table 3, Figure 3).

\section{Mortality of laryngeal cancer}

During the period of 2008-2012, there were 6,360 patients died from laryngeal cancer in China, accounted for $0.57 \%$ of cancer-related deaths. The crude mortality of laryngeal

Table 3 Trend of incidence of laryngeal cancer in China, 2003-2012

\begin{tabular}{|c|c|c|c|c|c|c|c|c|c|}
\hline \multirow[b]{2}{*}{ Year } & \multicolumn{3}{|c|}{ All } & \multicolumn{3}{|c|}{ Urban } & \multicolumn{3}{|c|}{ Rural } \\
\hline & $\begin{array}{c}\text { Both } \\
\left(1 / 10^{5}\right)\end{array}$ & $\begin{array}{c}\text { Male } \\
\left(1 / 10^{5}\right)\end{array}$ & $\begin{array}{c}\text { Female } \\
\left(1 / 10^{5}\right)\end{array}$ & $\begin{array}{c}\text { Both } \\
\left(1 / 10^{5}\right)\end{array}$ & $\begin{array}{c}\text { Male } \\
\left(1 / 10^{5}\right)\end{array}$ & $\begin{array}{c}\text { Female } \\
\left(1 / 10^{5}\right)\end{array}$ & $\begin{array}{c}\text { Both } \\
\left(1 / 10^{5}\right)\end{array}$ & $\begin{array}{c}\text { Male } \\
\left(1 / 10^{5}\right)\end{array}$ & $\begin{array}{c}\text { Female } \\
\left(1 / 10^{5}\right)\end{array}$ \\
\hline 2003 & 1.49 & 2.70 & 0.36 & 1.63 & 2.97 & 0.38 & 0.97 & 1.69 & 0.29 \\
\hline 2004 & 1.30 & 2.41 & 0.27 & 1.41 & 2.61 & 0.28 & 0.91 & 1.61 & 0.26 \\
\hline 2005 & 1.49 & 2.65 & 0.40 & 1.66 & 2.96 & 0.43 & 0.80 & 1.36 & 0.29 \\
\hline 2006 & 1.45 & 2.67 & 0.30 & 1.57 & 2.90 & 0.31 & 0.93 & 1.69 & 0.22 \\
\hline 2007 & 1.43 & 2.63 & 0.29 & 1.58 & 2.90 & 0.31 & 0.79 & 1.45 & 0.16 \\
\hline 2008 & 1.35 & 2.51 & 0.24 & 1.47 & 2.73 & 0.27 & 0.84 & 1.60 & 0.11 \\
\hline 2009 & 1.41 & 2.63 & 0.24 & 1.55 & 2.91 & 0.26 & 0.79 & 1.45 & 0.15 \\
\hline 2010 & 1.30 & 2.43 & 0.22 & 1.37 & 2.60 & 0.21 & 0.97 & 1.68 & 0.27 \\
\hline 2011 & 1.32 & 2.48 & 0.21 & 1.43 & 2.71 & 0.21 & 0.84 & 1.51 & 0.20 \\
\hline 2012 & 1.30 & 2.47 & 0.18 & 1.39 & 2.66 & 0.17 & 0.90 & 1.57 & 0.25 \\
\hline APC (\%) & -1.27 & -0.60 & -7.09 & -1.38 & -1.01 & -8.43 & -0.36 & -0.38 & -1.55 \\
\hline $95 \% \mathrm{Cl}(\%)$ & $-2.2,-0.3^{*}$ & $-1.6,0.4$ & $-10.3,-3.8^{*}$ & $-2.9,0.2$ & $-2.1,0.1$ & $-12.2,-4.4^{*}$ & $-2.3,1.6$ & $-2.1,1.4$ & $-5.9,3.0$ \\
\hline
\end{tabular}

$\mathrm{APC}$, annual percentage change; $95 \% \mathrm{Cl}, 95 \%$ confidence interval; ${ }^{*}, \mathrm{P}<0.05$ was considered statistically significant. 


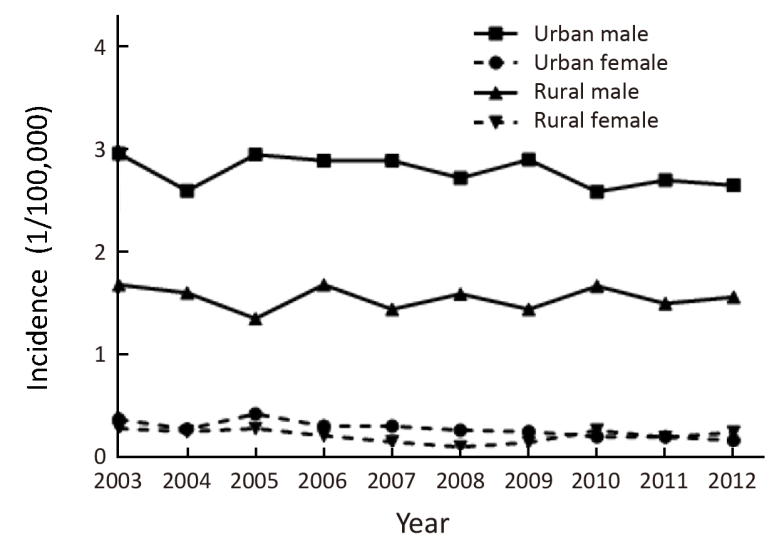

Figure 3 Trend of standardized incidence of laryngeal cancer in China, 2003-2012.

cancer was $1.01 / 100,000$ and it was the 21 st leading cause of cancer-related death in all cancer sites. Both the agestandardized mortality rates by Chinese population (ASMRC) and by World population (ASMRW) were $0.63 / 100,000$ and the cumulative mortality rate $(0-74$ years $)$ was $0.07 \%$.

The mortality was higher in males than in females. In males, the crude mortality, ASMRC and ASMRW were
$1.70 / 100,000,1.12 / 100,000$ and $1.12 / 100,000$, respectively, and in females, the rates were $0.30 / 100,000,0.17 / 100,000$ and $0.18 / 100,000$, respectively. The mortality rate in urban areas was higher than that in rural areas. In the former, the crude mortality, ASMRC and ASMRW were 1.10/100,000, $0.65 / 100,000$ and $0.65 / 100,000$, respectively, and in rural areas, they were $0.87 / 100,000,0.59 / 100,000$ and $0.60 / 100,000$, respectively. Laryngeal cancer was the $21 \mathrm{st}$ leading cause of cancer death in both urban and rural areas (Table 4).

The middle areas had a high crude mortality rate $(1.12 / 100,000)$, followed by the eastern $(0.99 / 100,000)$ and western areas $(0.88 / 100,000)$. After adjusted by age, the middle areas still had the highest mortality rate (ASMRC: 0.82/100,000, ASMRW: 0.82/100,000) and the mortality in western areas (ASMRC: 0.68/100,000, ASMRW: $0.69 / 100,000)$ was higher than that in eastern areas (ASMRC: 0.58/100,000, ASMRW: 0.58/100,000) (Table 4).

\section{Age-specific mortality rate of laryngeal cancer}

The mortality of laryngeal cancer in China was relatively low in age groups under 45 years and increased with age constantly. In males, the age-specific mortality curve before

Table 4 Laryngeal cancer mortality in China, 2008-2012

\begin{tabular}{llccccccc}
\hline Geographic areas & Gender & $\begin{array}{c}\text { Deaths } \\
(\times 1,000)\end{array}$ & $\begin{array}{c}\text { Mortality } \\
\left(1 / 10^{5}\right)\end{array}$ & $\begin{array}{c}\text { Ratio } \\
(\%)\end{array}$ & $\begin{array}{c}\text { ASMRC } \\
\left(1 / 10^{5}\right)^{*}\end{array}$ & $\begin{array}{c}\text { ASMRW } \\
\left(1 / 10^{5}\right)^{* *}\end{array}$ & $\begin{array}{c}\text { Cumulative rate } \\
0-74(\%)\end{array}$ & Rank \\
\hline \multirow{3}{*}{ All areas } & Both & 6.36 & 1.01 & 0.57 & 0.63 & 0.63 & 0.07 & 21 \\
& Male & 5.43 & 1.70 & 0.77 & 1.12 & 1.12 & 0.13 & 16 \\
& Female & 0.93 & 0.30 & 0.22 & 0.17 & 0.18 & 0.02 & 22 \\
Urban & Both & 4.22 & 1.10 & 0.61 & 0.65 & 0.65 & 0.07 & 21 \\
& Male & 3.67 & 1.90 & 0.86 & 1.19 & 1.19 & 0.13 & 16 \\
& Female & 0.55 & 0.29 & 0.21 & 0.15 & 0.15 & 0.02 & 23 \\
Rural & Both & 2.14 & 0.87 & 0.49 & 0.59 & 0.60 & 0.07 & 21 \\
& Male & 1.75 & 1.40 & 0.63 & 1.00 & 1.00 & 0.12 & 16 \\
Eastern areas & Female & 0.38 & 0.32 & 0.25 & 0.21 & 0.21 & 0.03 & 21 \\
& Both & 4.40 & 0.99 & 0.53 & 0.58 & 0.58 & 0.07 & 21 \\
& Male & 3.85 & 1.72 & 0.74 & 1.06 & 1.06 & 0.12 & 16 \\
Middle areas & Female & 0.55 & 0.25 & 0.18 & 0.13 & 0.13 & 0.01 & 23 \\
& Both & 1.48 & 1.12 & 0.70 & 0.82 & 0.82 & 0.10 & 21 \\
& Male & 1.17 & 1.72 & 0.88 & 1.33 & 1.33 & 0.16 & 14 \\
Western areas & Female & 0.31 & 0.49 & 0.40 & 0.34 & 0.34 & 0.04 & 21 \\
& Both & 0.48 & 0.88 & 0.57 & 0.68 & 0.69 & 0.07 & 21 \\
& Male & 0.41 & 1.49 & 0.75 & 1.20 & 1.21 & 0.13 & 17 \\
\hline
\end{tabular}

${ }^{*}$, age-standardized mortality rate by Chinese standard population in $2000 ;{ }^{\star \star}$, age-standardized mortality rate by Segi's population. 
70 years old in urban areas was similar to that in rural areas, and in groups aged over 70 years old, the mortality was higher in urban areas than in rural areas. In females, agespecific mortality curves were similar in both urban and rural areas. The mortality rate of laryngeal cancer of females in middle areas was higher than that in eastern areas and western areas in the groups of 40-79 years old, but similar for males. The mortality rate peaked in the $80-85+$ age groups in overall areas and urban areas. In rural areas, the mortality rate in the $85+$ age group was the highest among all age groups. In eastern areas, middle areas and western areas, the mortality rate peaked in the $80-84$, 75-79 and 85+ age groups, respectively (Figure 4, 5).

\section{Mortality trend of laryngeal cancer}

The mortality rate of laryngeal cancer decreased annually from 2003 to 2012 in China. It decreased by $13.92 \%$ (11.97\% for males and $34.78 \%$ for females, $16.67 \%$ for

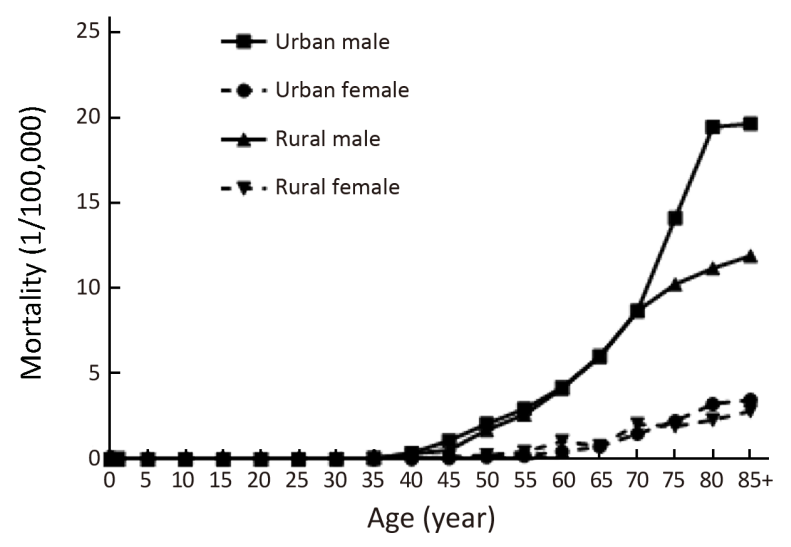

Figure 4 Age-specific mortality of laryngeal cancer in urban and rural areas in China, 2008-2012.

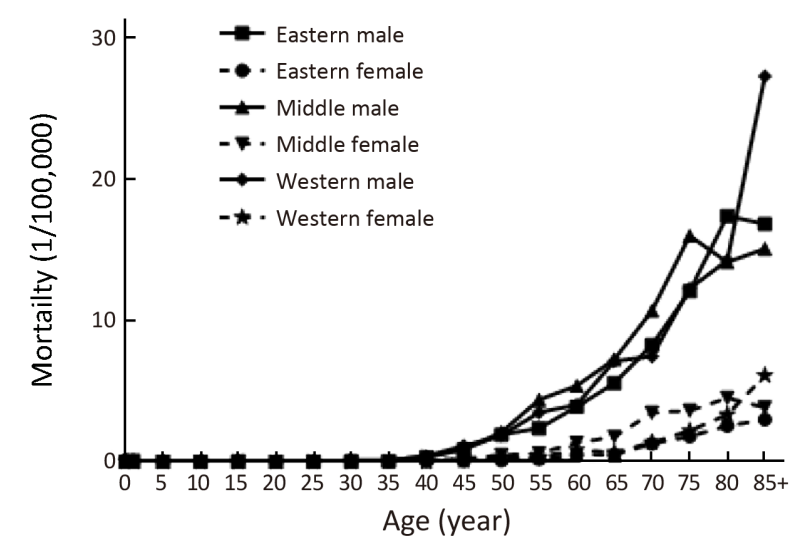

Figure 5 Age-specific mortality of laryngeal cancer in different regions in China, 2008-2012. urban areas and $4.84 \%$ for rural areas) compared with 2003. The descent range of mortality rate in females was bigger than that in males, and the descent range in urban areas was bigger than that in rural areas. The time change trend of the mortality rate in females was statistically significant (APC: $-5.18 \%$; 95\% CI: $-9.0 \%,-1.2 \%$ ) $(\mathrm{P}<0.05)$, and the trends in males and total were not statistically significant, with the APC of $-0.98 \%$ (95\% CI: $-2.6 \%, 0.7 \%)$ for males and $-1.15 \%$ (95\% CI: $-2.5 \%$, $0.2 \%)$ in total. In urban areas, the APC of the mortality rate in total, males and females was $-1.22 \%(95 \% \mathrm{CI}$ : $-3.0 \%, 0.7 \%),-1.47 \%$ (95\% CI: $-3.3 \%, 0.4 \%)$ and $-3.88 \%$ (95\% CI: $-8.9 \%, 1.5 \%$ ), respectively, and the time trend of laryngeal cancer mortality rate was not statistically significant. In the rural areas, the APC of the total was $-2.55 \%$ (95\% CI: $-6.9 \%, 1.9 \%)$. According to gender stratification, the APC of the mortality rate in males was $-0.96 \%$ (95\% CI: $-4.7 \%, 2.9 \%)$ and that in females was $-4.96 \%$ (95\% CI: $-11.6 \%, 2.2 \%)$. The time change trends of the mortality rate in males, females and both sexes were not statistically significant (Table 5, Figure 6).

\section{Discussion}

Laryngeal cancer is a malignant tumor originating from the laryngeal mucosal epithelium. Squamous cell carcinoma (SCC) is the most common type of laryngeal cancer, accounting for about $98 \%$ of all new cases. Smoking, alcohol drinking, sustained inhalation of harmful substances and infection of papillomavirus may lead to laryngeal cancer (7). The laryngeal cancer was accounting for $1 \%-5 \%$ of overall new cancer cases and ranked third in the cancers of ear-nose-throat and occurred more often in middle-aged patients and in males (8-10).

Based on the data provided in this study, the laryngeal cancer was the 21 st most common cancer and 21 st leading cause of cancer-related death in China. The incidence of laryngeal cancer was 7.68 times higher in males than in females. Similar with our results, the ratio of male to female was 7.8:1 in the world (11), 7:1 in Spain (12), 10.5:1 in Kazakhstan from 1999 to 2009 (13), 7.22:1 in China from 2003 to 2007 (14). The reasons for higher rates in males probably reflect etiologic factors related to sex hormones and other risk factor exposures like smoking, alcohol drinking, and so on.

In China, both incidence and mortality of laryngeal cancer were higher in urban areas than in rural areas. Laryngeal cancer in urban areas was approximately $40 \%$ higher than those in rural areas for age-standardized 
Table 5 Trend of mortality of laryngeal cancer in China, 2003-2012

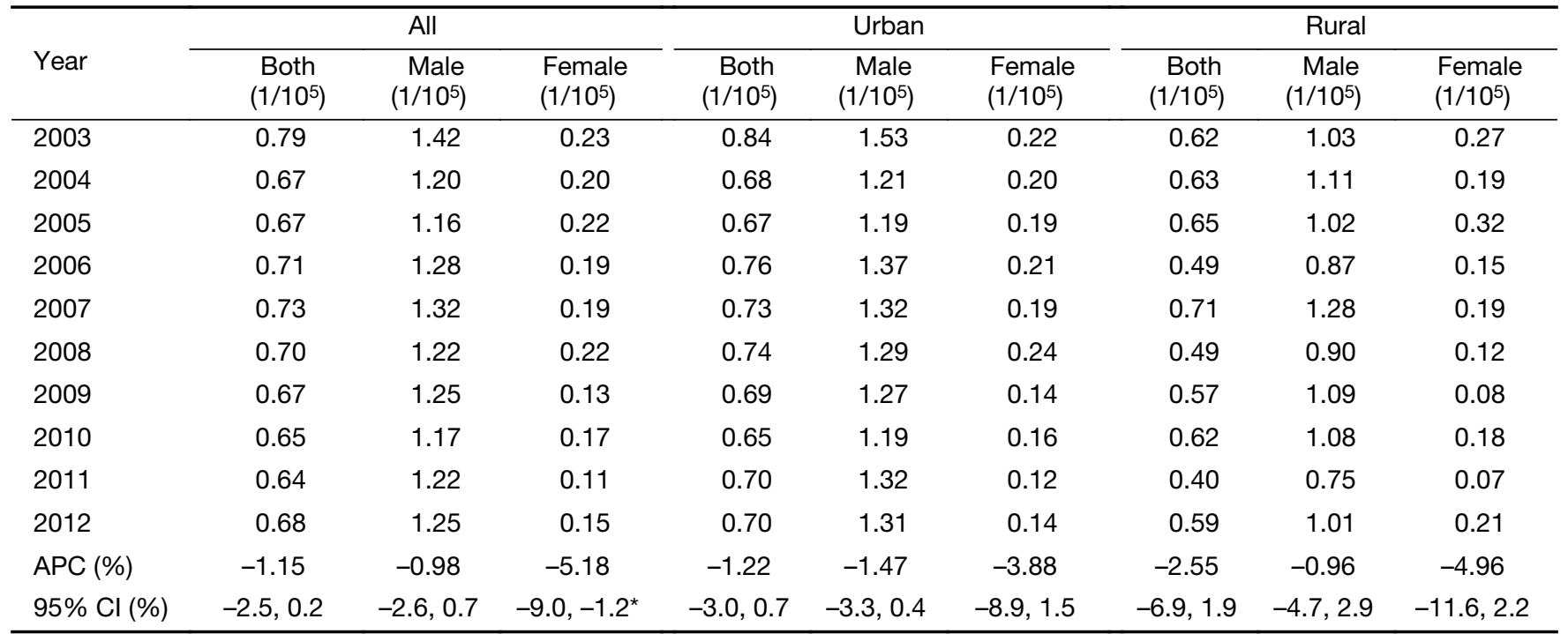

$\mathrm{APC}$, annual percentage change; $95 \% \mathrm{Cl}, 95 \%$ confidence interval; * $\mathrm{P}<0.05$ was considered statistically significant.

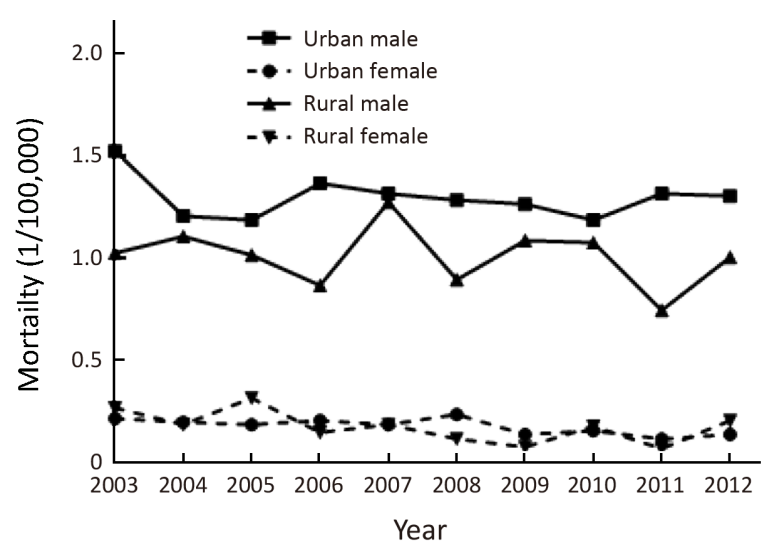

Figure 6 Trend of mortality of laryngeal cancer in China, 2003-2012.

incidence rate and $10 \%$ higher for age-standardized mortality rate. The reason may be more serious air and water pollution in urban areas than in rural areas. Zhang et al. (15) showed that the incidence of laryngeal cancer in Liaoning province of China was higher than that in rural areas, and the incidence of urban cancer was 2.3 times as high as that in rural areas and other study also showed the same result (16).

There were regional disparity in both incidence and mortality of laryngeal cancer in China. The incidence rate in eastern areas and western areas was similar, and lower than middle areas. The middle areas had the highest mortality rate, followed by eastern areas and western areas. Much of this disparity may be due to the natural geographical environment, the different socioeconomic levels, lifestyles and conditions of local health care (17-19).

The decreased trend in incidence and mortality rate of laryngeal cancer was found in China from 2003 to 2012. The mortality rate decreased great than the incidence rate in urban areas. The incidence rates of laryngeal cancer in females and in total showed statistical significance decline trend and the time change trend in males was not statistically significant in China. The time change trend of the mortality rate had statistical significance only in females. Meanwhile in rural areas of China, the time change trends of both the incidence and mortality of laryngeal cancer were not statistically significant. The main reason may be the disparity of the medical resources between the urban areas and rural areas. There are better quality of cancer care, enough medical treatment, high educated degree and earlier screening program for patients living in urban China. The government should balance the medical resources and take effective measures to bridge the gap (20).

\section{Conclusions}

Laryngeal cancer is a relatively uncommon cancer in China and showed a declined trend of rate in recent years. There are disparities of incidence rate in genders, age groups and different areas. Appropriate targeted prevention, early detection and treatment programs can be carried out to control the local burden of laryngeal cancer. 


\section{Acknowledgements}

We thank the Bureau of Disease Control, National Health and Family Planning Commission of the People's Republic of China for their support to this study. We gratefully acknowledge all the population-based cancer registries for the effort they put in data collection, data sorting, data verification and database creation. The authors take full responsibility for analyses and interpretation of these cancer registry data.

\section{Footnote}

Conflicts of Interest: The authors have no conflicts of interest to declare.

\section{References}

1. Chen $\mathrm{W}$, Zheng R, Zhang S, et al. Cancer incidence and mortality in China, 2013. Cancer Let 2017; 401:63-71.

2. Torre LA, Bray F, Siegel RL, et al. Global cancer statistics, 2012. CA Cancer J Clin 2015;65:87-108.

3. National Cancer Center. Chinese Guideline for Cancer Registration. Beijing: People's Medical Publishing House, 2016.

4. Curade MP, Edwards B, Shin HR, et al. Cancer incidence in five continents. Volume IX. IARC Sci Publ 2008:1-837.

5. Chen W, Sun K, Zheng R, et al. Cancer incidence and mortality in China, 2014. Chin J Cancer Res 2018;30:1-12.

6. Di J, Rutherford S, Chu C. Review of the cervical cancer burden and population-based cervical cancer screening in China. Asian Pac J Cancer Prev 2015;16:7401-7.

7. Kara E, Cetik MF, Tuncer U, et al. A rare association of leiomyosarcoma with squamous cell carcinoma of the larynx: two cases. J Laryngol Otol 2013;127: 828-31.

8. Du L, Li H, Zhu C, et al. Incidence and mortality of laryngeal cancer in China, 2011. Chin J Cancer Res 2015;27:52-8.

9. Chen W, Zheng R, Zuo T, et al. National cancer incidence and mortality in China, 2012. Chin J Cancer Res 2016;28:1-11.

10. Chen $\mathrm{W}$, Zheng $\mathrm{R}$, Zhang $\mathrm{S}$, et al. Cancer incidence and mortality in China in 2013: an analysis based on urbanization level. Chin J Cancer Res 2017;29:1-10.

11. Xie $Y$, Min $H$, Zeng $Z$, et al. Investigation of the relationship between laryngeal carcinoma and sex hormone receptor, and the determination of preoperative-postoperative serum sex hormone levels. Ai Zheng (in Chinese) 1994;13:432-4.

12. de Souza DL, Pérez MM, Curado MP. Gender differences in the incidence of laryngeal and hypopharyngeal cancers in Spain. Cancer Epidemio1 2011;35:328-33.

13. Igissinov $\mathrm{N}$, Zatoskikh $\mathrm{V}$, Moore MA, et al. Epidemiological evaluation of laryngeal cancer incidence in Kazakhstan for the years 1999-2009. Asian Pac J Cancer Prev 2013;14:3969-74.

14. Du L, Mao W, Chen W, et al. Analysis of incidence and mortality of laryngeal cancer in China from 2003 to 2007. Zhonghua Liu Xing Bing Xue Za Zhi (in Chinese) 2012;33:395-8.

15. Zhang K, Fei S, Chen Z, et al. Relationship between air pollution and incidence of laryngeal cancer in industrial cities of Liaoning province. Zhonghua Er Bi Hou Tou Jing Wai Ke Za Zhi (in Chinese) 1990; 25:240-2.

16. Gong L, Li L, Gu J, et al, Analysis of the trend of laryngeal cancer in west Liaoning between 2003 and 2012. Analysis of the trend of laryngeal cancer in west Liaoning between 2003 and 2012. Zhongguo Lao Nian Xue Za Zhi (in Chinese) 2015;35:3113-4.

17. Siegel RL, Miller KD, Jemal A. Cancer statistics, 2018. CA Cancer J Clin 2018;68:7-30.

18. Taguchi T, Nishimura G, Takahashi $M$, et al. Treatment results and prognostic factors for advanced squamous cell carcinoma of the larynx treated with concurrent chemoradiotherapy. Cancer Chemother Pharmacol 2013;72:837-43.

19. Chen W, Zheng R, Baade PD, et al. Cancer statistics in China, 2015. CA Cancer J Clin 2016;66:115-32.

20. Zhang SS, Xia QM, Zheng RS, et al. Laryngeal cancer incidence and mortality in China, 2010. J Cancer Res Ther 2015;11 Suppl 2:C143-8.
Cite this article as: Liu Y, Zhao Q, Ding G, Zhu Y, Li W, Chen $W$. Incidence and mortality of laryngeal cancer in China, 2008-2012. Chin J Cancer Res 2018;30(3):299-306. doi: 10.21147/j.issn.1000-9604.2018.03.02 